\title{
A tale of two mitochondrial channels, MAC and PTP, in apoptosis
}

\author{
Kathleen W. Kinnally • Bruno Antonsson
}

Published online: 6 February 2007

(C) Springer Science + Business Media, LLC 2007

\begin{abstract}
The crucial step in the intrinsic, or mitochondrial, apoptotic pathway is permeabilization of the mitochondrial outer membrane. Permeabilization triggers release of apoptogenic factors, such as cytochrome $c$, from the mitochondrial intermembrane space into the cytosol where these factors ensure propagation of the apoptotic cascade and execution of cell death. However, the mechanism(s) underlying permeabilization of the outer membrane remain controversial. Two mechanisms, involving opening of two different mitochondrial channels, have been proposed to be responsible for the permeabilization; the permeability transition pore (PTP) in the inner membrane and the mitochondrial apoptosis-induced channel (MAC) in the outer membrane. Opening of PTP would lead to matrix swelling, subsequent rupture of the outer membrane, and an unspecific release of intermembrane proteins into the cytosol. However, many believe PTP opening is a consequence of apoptosis and this channel is thought to principally play a role in necrosis, not apoptosis. Activation of MAC is exquisitely regulated by Bcl-2 family proteins, which are the sentinels of apoptosis. MAC provides specific pores in the outer membrane for the passage of intermembrane proteins, in particular cytochrome $c$, to the cytosol. The electrophysiological characteristics of MAC are very similar to Bax channels and depletion of Bax
\end{abstract}

K. W. Kinnally $(\bowtie)$

Department of Basic Sciences, New York University

College of Dentistry,

345 East 24th Street, New York, NY 10010, USA

e-mail:kck1@nyu.edu

B. Antonsson

Serono Pharmaceutical Research Institute,

14, Chemin des Aulx,

CH-1228 Plan-les Ouates, Geneva, Switzerland significantly diminishes MAC activity, suggesting that Bax is an essential constituent of MAC in some systems. The characteristics of various mitochondrial channels and Bax are compared. The involvement of MAC and PTP activities in apoptosis of disease and their pharmacology are discussed.

Keywords MAC: Mitochondrial apoptosis-induced channel - PTP: Permeability transition pore - Patch clamp . Pharmacology $\cdot \mathrm{Bax} \cdot \mathrm{Bcl}-2 \cdot$ Apoptosis

Controlling the number of cells in multi-cellular organisms is an essential feature from the early embryonic stage, to ensure correct development of organs, and throughout the adult life, to keep a correct balance and provide the body with the cells required at different stages in life. This can be achieved through regulation of cell proliferation, increasing or restricting the creation of new cells, or through the elimination of old and damaged cells or cells that are no longer needed, e.g., activated immune cells. The ultimate regulation is mostly a finely controlled combination of the two processes. The elimination of cells is a tightly regulated process initiated by specific signals, which trigger the targeted cells to commit suicide and die by apoptosis. During this process, DNA fragments into a characteristic degradation pattern and the cell body is dispersed as apoptotic bodies that are eliminated by phagocytosis. Although the end result is the same, there are several means of achieving apoptosis (reviewed in [112]). The upstream signaling cascades triggering apoptosis are very different depending on the initiating stimuli and the targeted cells to be eliminated. However, as most things in life, this essential process also has a dark side. Apoptosis has also been shown to be associated with a large number of pathological conditions. 


\section{Apoptosis signaling pathways}

Two classical apoptosis signaling cascades have long been known and they are the receptor pathway and the mitochondrial pathway. The extrinsic pathway, also known as the receptor pathway, is activated through binding of ligands to cell membrane receptors, such as Fas, TNF or the TRAIL receptors [13]. Upon ligand binding, the receptors oligomerize, which triggers the recruitment of adaptor proteins and procaspases, like caspase 8 or 10 , to the receptor complex. The caspases are activated in the complex and the active caspases further amplify the apoptosis signaling cascade through activation of downstream effector caspases like caspase 3 or 7 .

The intrinsic, or mitochondrial pathway, is a complex pathway with mitochondria as central gateway controllers and the multi-domain pro-apoptotic proteins, Bax, Bak and may be Bok, as executioners. The signaling cascades upstream of the mitochondria are very diversified and still not well understood at the molecular level. It is clear that the molecules upstream, like the $\mathrm{BH} 3$-only proteins, capture various apoptosis stimuli or signals and subsequently activate the multi-domain pro-apoptotic proteins. How this activation takes place and which signals activate $\mathrm{BH} 3$-only proteins, often remains to be elucidated. Whether solely BH3-only proteins can activate the multi-domain proteins is also unclear; there might be other activating partners. Activation of the intrinsic pathway involves release of pro-apoptotic factors, like cytochrome $c$, Smac/Diablo, Endonuclease G, and AIF, from the mitochondrial intermembrane space, which amplifies the apoptotic cascades to the final destruction of the cell $[1,2,4,9,14]$.

The apoptosome/caspase 9 pathway is a signaling pathway downstream of the mitochondrion that has been well described. The release of cytochrome $c$ triggers formation of the apoptosome complex and activation of caspase 9 [15]. However, this does not appear to be the only pathway that can be activated through permeabilization of the outer mitochondrial membrane. Two proteins, Endonuclease $G$ and AIF, released after permeabilization of the outer membrane, can induce apoptosis in a caspase-independent manner [16, 17]. What governs whether permeabilization of the outer mitochondrial membrane activates one or the other downstream pathways still remains unknown.

The extrinsic and intrinsic pathways initially appeared to be independent. However, it is now clear that a crosstalk exists between the two pathways, which is mediated by the BH3-only protein Bid [18]. After activation of the extrinsic pathway in some cell types, caspase 8 cleaves Bid to form t-Bid, which facilitates Bax activation, oligomerization, and insertion into the mitochondrial outer membrane, i.e., MAC formation. Thus the t-Bid generated by the extrinsic pathway

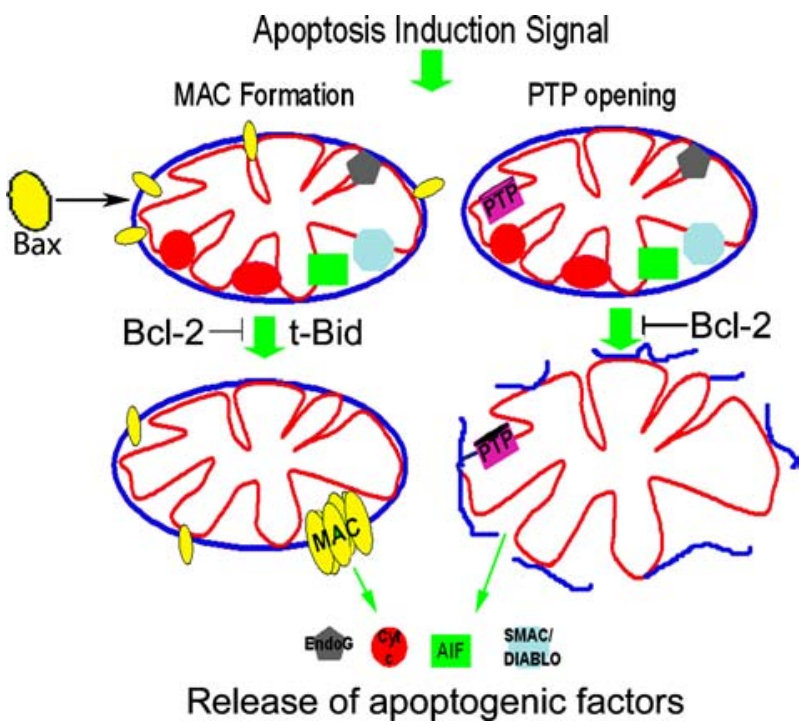

Fig. 1 Models for permeabilization of the mitochondrial outer membrane. Cartoon illustrates two putative mechanisms for release of proapoptogenic factors like cytochrome $c(\mathrm{Cyt} c)$, Endonuclease $\mathrm{G}$ (Endo G), SMAC/Diablo and AIF from the intermembrane space. Left side Upon induction of apoptosis, the multi-domain proapoptotic protein Bax translocates to the outer membrane and both Bax and Bak (yellow) are activated. Bax and/or Bak oligomerize to form MAC, the channel through which proapoptotic factors pass into the cytosol. BH-3 only proteins like t-Bid facilitate and the anti-apoptotic proteins like Bcl-2 block these events. Right side PTP (purple) opens to permeabilize the inner membrane, depolarize the mitochondria, and allow passage of $<1500 \mathrm{MW}$ solutes. The matrix space swells, ruptures the outer membrane, and spills pro-apoptogenic factors into the cytosol. Anti-apoptotic proteins, like Bcl-2, block PTP opening. $\dashv$ indicates blockade

activates the intrinsic pathway to ensure complete execution of apoptosis.

There are two candidate pathways that diverge at the mechanism responsible for the release of mitochondrial mediators of cell death. Both mechanisms result in permeabilization of the outer membrane but do so by activation of two distinct channels. These channels are the permeability transition pore PTP in the inner membrane and the mitochondrial apoptosis-induced channel MAC in the outer membrane (Fig. 1).

Opening of the PTP in the inner membrane causes depolarization of the membrane potential and swelling of the matrix space. This swelling ruptures the outer membrane, and spills cytochrome $c$ and other pro-apoptotic proteins into the cytosol [11, 12, 19-21]. Recent studies of the PTP regulator cyclophilin have raised doubts about the importance of PTP in triggering apoptosis (see below). Furthermore, cytochrome $c$ release can occur in the absence of mitochondrial depolarization and without loss of outer membrane integrity. These observations indicate that, instead of rupturing, a more selective mechanism of permeabilization is operating, like the formation of a pore in the outer membrane [14, 22-26]. 
MAC forms in the mitochondrial outer membrane early in apoptosis and directly provides a pathway for the release of cytochrome $c$ from the intermembrane space to the cytosol. MAC is exquisitely regulated by Bcl-2 family proteins and the multi-domain pro-apoptotic protein Bax and Bak are putative components of this channel [3, 8, 10, 23, 24, 27]. At this time, the complete molecular identity of MAC is not known and studies suggest MAC may contain additional components [28].

Transient opening of MAC and PTP may alone or in combination, depending on cell type and death stimulus, result in remodeling of the cristae and maximal cytochrome $c$ release to amplify the death signal and ensure completion of apoptosis $[10,29]$. Both channels are subject to regulation by Bcl-2 family proteins [23, 24, 30, 31]. The focus of this review is a comparison of MAC and PTP and their possible roles in apoptosis.

\section{Characterization of Bax channels}

The three-dimensional structures of the Bcl-2 family proteins show close resemblance to bacteria toxins that form channels [32-34]. The proteins possess two central hydrophobic helixes surrounded by amphipathic helixes. It is somewhat surprising that this structural arrangement appears to be conserved in both pro- and anti-apoptotic members of the Bcl-2 family. Consistent with this common structure, both the antiand the pro-apoptotic proteins have channel-forming activity in vitro, although the channel forming conditions differ [35-37]. In normal healthy cells, Bax is present as a cytosolic, monomeric protein, which doesn't have channel-forming capacity [38]. However, once activated, Bax translocates to mitochondria; it is then inserted into the outer membrane and oligomerizes to form large channels [39].
Activation of Bax is associated with conformational changes in both the $\mathrm{C}$ - and the N-terminal parts of the protein $[34,40,41]$. In vitro activation can be induced by either exposing the protein to detergents, like Triton X-100 or octyl glycoside, or through interactions with t-Bid, a natural activator of the multi-domain proteins [38, 39, 42]. Oligomeric Bax forms slightly cation-selective, voltage-independent channels with a variable conductance from a few $\mathrm{pS}$ of up to several $\mathrm{nS}[35,42]$. The diameter estimated for the Bax channels from the peak conductance of $1-5.4 \mathrm{nS}$ is $2.7-$ $5.4 \mathrm{~nm}$, indicating many Bax channels could easily allow the passage of $\sim 3 \mathrm{~nm}$ cytochrome $c$ (see Table 1) [23, 43]. Bax channels have been shown to increase the permeability of liposomes to small molecules, like 5,6-carboxyfluorescein and fluorescein-dextran $[39,44]$. One study showed that Bax tetramers inserted into liposomes and formed channels large enough to allow the release of cytochrome $c$, which demonstrated that Bax alone is able of creating channels large enough for the passage of mitochondrial proteins through membranes [44]. Bax channels and the release of various compounds from liposomes are blocked by the anti-apoptotic proteins Bcl-2 and Bcl-xL [35]. Furthermore, cytochrome $c$ and RNAse $\mathrm{A}$ induce Type 1 and Type 2 effects in recombinant Bax channels, while hemoglobin has no effect [24]. Type 1 effects are a reversible 5-50\% decrease in conductance that is voltage dependent. Type 2 effects are a larger decrease in conductance that is not reversible or voltage dependent.

In contrast to Bax, Bak is always found in the mitochondria. While Bak need not translocate, its activity is presumably induced through oligomerization of Bak molecules already in the membrane [45]. Extensive in vitro studies of Bak have been hampered mainly by difficulties in obtaining sufficient quantities of purified recombinant protein. While intriguing, the role of the mitochondrial fission
Table 1 Comparison of the activities of Bax and Mitochondrial channels

\begin{tabular}{|c|c|c|c|c|c|}
\hline & $\mathrm{PTP}^{\mathrm{a}}$ & MAC $^{\mathrm{b}}$ & $\mathrm{hBax}^{\mathrm{c}}$ & $\mathrm{TOM}^{\mathrm{d}}$ & VDAC $^{d}$ \\
\hline Peak conductance $(\mathrm{nS})$ & $1.1 \pm 0.1$ & $1-5$ & $1.0-5.4$ & $0.7 \pm 0.1$ & $0.7 \pm 0.1$ \\
\hline Transition size (nS) & $\begin{array}{l}0.3-1.0 \\
0.34 \pm .05 \\
0.74 \pm .01\end{array}$ & $\begin{array}{l}0.3-2.0 \\
1.5 \pm 0.2\end{array}$ & $0.1-1.0$ & $0.34 \pm 0.03$ & $0.36 \pm 0.04$ \\
\hline Ion selectivity & Sl. Cation & Sl. Cation & Sl. Cation & Sl. Cation & Anion \\
\hline $\mathrm{P}_{\mathrm{K}} / \mathrm{P}_{\mathrm{Cl}}$ & 7 & $4.7 \pm 1.3$ & $6.8 \pm 1.0$ & $3.6 \pm 0.8$ & $0.7 \pm 0.1$ \\
\hline Voltage dependent & Yes & No & No & Yes & Yes \\
\hline Pore diameter $\mathrm{e}^{\mathrm{e}}(\mathrm{nm})$ & $2.8 \pm 0.1$ & $2.7-6.0$ & $2.7-6.2$ & $2.2 \pm 0.1$ & $2.2 \pm 0.05$ \\
\hline
\end{tabular}

${ }^{\text {aPTP }}$ of MDA231 breast cells [30].

${ }^{\mathrm{b}}$ Mammalian MAC of apoptotic HeLa and FL5.12 cells [23-25].

${ }^{\mathrm{c}}$ Recombinant human Bax [25].

${ }^{\mathrm{d}}$ Mammalian TOM and VDAC of apoptotic FL5.12 cells [25].

${ }^{\mathrm{e}}$ Calculated from peak transition size assuming $7 \mathrm{~nm}$ pore length [99] 
and fusion machinery in activation and/or oligomerization of Bax and/or Bak as well as cytochrome $c$ release are yet to be determined [46].

In order to study Bax channel activity and its relevance to apoptosis, specific Bax channel blockers were developed. The action of these blockers was tested in both in vitro and in vivo apoptosis model systems [47] (see Fig. 4 and discussion below).

\section{Electrophysiological characterization of MAC}

MAC was first detected in patch-clamp experiments on mitochondria isolated from apoptotic FL5.12 cells $12 \mathrm{~h}$ after withdrawal of the growth factor interleukin-3 (IL-3) [25] and has since been studied in several systems [23, 24, 27, 29]. MAC is a heterogeneous high-conductance channel with multiple sub-conductance levels. While MAC can spontaneously and reversibly close, this channel typically occupies the open state with mean open times of seconds. MAC activity is remarkably similar to the channel activity of recombinant Bax

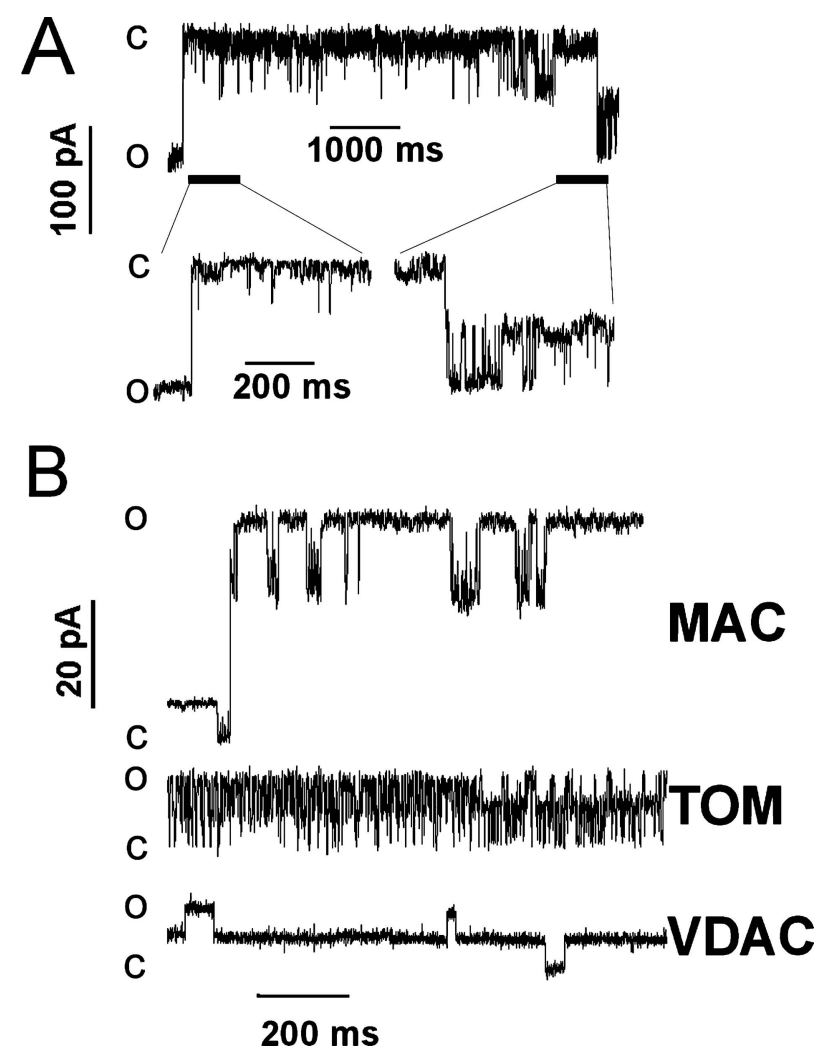

Fig. 2 Comparison of the activities of MAC, VDAC, and TOM channels. (A) Single-channel current traces reveal MAC activity with $3 \mathrm{nS}$ peak transition size and predominant transitions of $1.5 \mathrm{nS}$ at $-40 \mathrm{mV}$. (B) Current traces of single channels at $20 \mathrm{mV}$ show MAC character is distinct from TOM channel and VDAC. $\mathrm{O}$ and $\mathrm{C}$ indicate open and closed conductance levels. Patching media was symmetrical $150 \mathrm{mM}$ $\mathrm{KCl}, 5 \mathrm{mM}$ HEPES-KOH/pH 7.4. Current traces were low pass filtered at $2 \mathrm{kHz}$ (5 kHz sampling). Modified from Fig. 2 of Pavlov et al. (2001) J Cell Biol 155:725-31 [25] recorded in both planar bilayers and liposomes. As described below, studies have demonstrated that Bax is a component of MAC in apoptotic HeLa and MEF cell lines. In contrast, the activity of MAC is significantly different from the constitutive channels of the mitochondrial outer membrane, TOM and VDAC. The single channel parameters of peak conductance, transition size, selectivity, and voltage dependence for these channels are shown in Table 1 and illustrated by the current traces of Fig. 2.

Efforts to characterize MAC have focused on the activity detected at the time of cytochrome $c$ release. Like Bax, this channel is slightly cation-selective, which is consistent with MAC's putative role in releasing the cationic protein cytochrome $c$. MAC is typically a voltage-independent channel [23-25, 27], although it has also been reported to occupy less than peak conductance at higher potentials [29]. Conductance is a measure of channel size and the conductance of MAC at the time of cytochrome $c$ release is both variable and high [23, 24]. The mean conductance of MAC of apoptotic HeLa and FL5.12 cells is 3.3 and $4.5 \mathrm{nS}$, respectively [23-25]. The polymer exclusion method shows $4 \mathrm{nS}$ MAC channels are permeable to 10 and $17 \mathrm{kDa}$, but not 45 and $71 \mathrm{kDa}$ Dextran [24]. This approach indicates that channels with a conductance between 1.5 and $5 \mathrm{nS}$ have pore sizes in the range of 3.3-6.0 nm.

The release of cytochrome $c$, which has a diameter of $\sim 3 \mathrm{~nm}$ [43], occurs earlier than that of other pro-apoptotic factors in some systems [48-50]. However, there are reports of cytochrome $c$ release at the same time as several higher MW pro-apoptotic factors including SMAC/Diablo and AIF [49-53]. Importantly, MAC has only been characterized early in apoptosis at the time of cytochrome $c$ release. It is likely that the mean pore size of MAC increases as apoptosis progresses possibly by incorporation of additional $\mathrm{Bax}$ and/or Bak molecules into the MAC channel complex, or perhaps by other, as yet unidentified, components.

Cytochrome $c$ and RNAase A cause a reversible voltagedependent decrease in MAC conductance, (Table 2, Type 1 effect) consistent with their partitioning into the pore [24]. These molecules have the same effects on pure Bax channels [23]. While hemoglobin has no effect, cytochrome $c$ and RNAase can also cause a Type 2 effect, which is an irreversible, voltage-independent closure of MAC. Importantly, and similar to Bax channel activity, over-expression of Bcl-2 blocks formation of MAC and release of cytochrome $c$ [25].

\section{Bax and Bak are functionally redundant with respect to MAC}

MAC and Bax channel activities appear to be identical when examined with electrophysiological techniques; they are very large conductance channels that are essentially 
Table 2 Some

Pharmacological effectors of MAC/Bax channels, and PTP

${ }^{\mathrm{a}} \mathrm{IC}_{50}$.

${ }^{\mathrm{b}}$ Concentration used.

${ }^{\mathrm{c}} \mathrm{Nd}$ not determined.

\begin{tabular}{llllll}
\hline Agent & Apoptosis & \multicolumn{2}{r}{ MAC/Bax $\mu \mathrm{M}^{\mathrm{a}}$} & \multicolumn{2}{c}{ PTP $\mu \mathrm{M}^{\mathrm{b}}$} \\
\hline Cyclosporine A [11, 27, 76] & Blocks & No effect & - & Blocks & 0.5 \\
Bci1 [47, 100] & Blocks & Blocks & 0.8 & $\mathrm{Nd}^{3}$ & - \\
Bci2 [47] & Blocks & Blocks & 0.9 & $\mathrm{Nd}$ & - \\
2-propanol derivatives [100] & - & Blocks & $0.3-2.4$ & $\mathrm{Nd}$ & - \\
Dibucaine [27, 87] & $\mathrm{Nd}$ & Blocks & 39 & Blocks & 500 \\
Propranolol [27, 88] & $\mathrm{Nd}$ & Blocks & 52 & Blocks & 700 \\
Trifluoperazine [27, 87] & - & Blocks & 0.9 & Blocks & 500 \\
Amiodarone [88] & - & Nd & - & Blocks & 4 \\
Lidocaine [27] & Nd & No effect & - & Nd & - \\
Cytochrome $c$ [24] & Induces & Pore permeation 100 & Nd & - \\
RNAase A [24] & Nd & Pore permeation & 100 & Nd & - \\
Ro5-4864 [101] & Blocks & Nd & - & Blocks & 0.07 \\
PPIX [101] & Nd & Nd & - & Block/open & $.02 / .24$ \\
\hline
\end{tabular}

voltage independent, have many substates, and a variable peak conductance. These functional similarities suggest Bax and MAC constitute the same entity. Furthermore, the initial time of MAC detection coincides with the time of translocation of $\mathrm{Bax}$ to the mitochondrial membrane. Hence, the possibility that Bax is a component of MAC was examined with biochemical and molecular approaches.

Immunoprecipitation of oligomeric Bax results in a depletion of MAC activity from lysates of staurosporine-treated HeLa cells. Bax oligomerizes in the outer membrane following its translocation to mitochondria, which is coincident with cytochrome $c$ release [22, 23, 28, 54]. MAC activity is present in total mitochondrial lysates and the activity co-elutes with chromatography fractions containing oligomeric Bax, but not monomeric Bax (Fig. 3) [23]. Importantly, Bax antibodies raised against an N-terminal epitope of the protein deplete MAC activity from solubilized mitochondrial fractions [23]. The N-terminal epitope is not accessible in monomeric Bax but is exposed following activation of Bax. Therefore, these antibodies selectively precipitate oligomeric, not monomeric, Bax [40, 41]. The concomitant loss of MAC activity upon immunoprecipitation of oligomeric Bax indicates that Bax is a component of MAC in this system.

The relationship between MAC and the multi-domain proapoptotic proteins Bax and Bak was further examined using molecular approaches. Previous studies using single and double knock out cell lines for Bax and Bak showed these two proteins are functionally redundant with respect to their roles in apoptosis (see $[1,2,5,9]$ for review). That is, cytochrome $c$ release occurs in Bax and Bak single knockout cells but not in the double Bax/Bak knockout cells during staurosporine treatment (Fig. 3) [23, 26]. Similarly, MAC is detected in single knockout, but not the double knockout cell lines during apoptosis (Fig. 3). As immunodepletion studies showed Bax is a component of MAC in apoptotic HeLa cells, these data indicate that Bak can replace Bax as a structural com- ponent of MAC in Bax deficient cells. That is, Bax and Bak are functionally redundant with respect to MAC.

Because of these findings, recombinant Bax has become a valuable model system for studying both MAC and cytochrome $c$ release. Unfortunately, difficulties in producing recombinant forms of other multi-domain pro-apoptotic proteins like Bak have excluded their electrophysiological characterization and usefulness as model systems.

\section{Electrophysiological characterization of PTP}

While MAC directly permeabilizes the mitochondrial outer membrane to release cytochrome $c$, the permeability transition pore PTP indirectly releases apoptogenic factors from the intermembrane space. Opening of PTP causes depolarization and swelling of the matrix space. Since the inner membrane has a surface area several times that of the outer membrane, swelling of the matrix space results in rupture of the outer membrane and a spilling of intermembrane space proteins into the cytosol.

The PTP was originally described in swelling experiments (detected as changes in scatter) on isolated mitochondria in landmark studies more than 25 years ago (Fig. 4) [55-58]. Opening of the PTP leads to an increase in permeability of the mitochondrial inner membrane for solutes up to $1.5 \mathrm{kDa}$ and is typically studied by changes in light scattering, i.e., absorbance. PTP can often be reversibly closed by removal of calcium with EGTA or by the addition of cyclosporine A, magnesium or ADP. The PTP can be activated by a myriad of effectors including calcium plus phosphate and reactive oxygen species. This channel has been implicated in the apoptotic cascade as a means of releasing cytochrome $c$ from mitochondria [11, 12, 19, 29].

The connection between the PTP and channel activities recorded directly from the inner membrane (by patch clamping mitoplasts) was made over a few years by several 


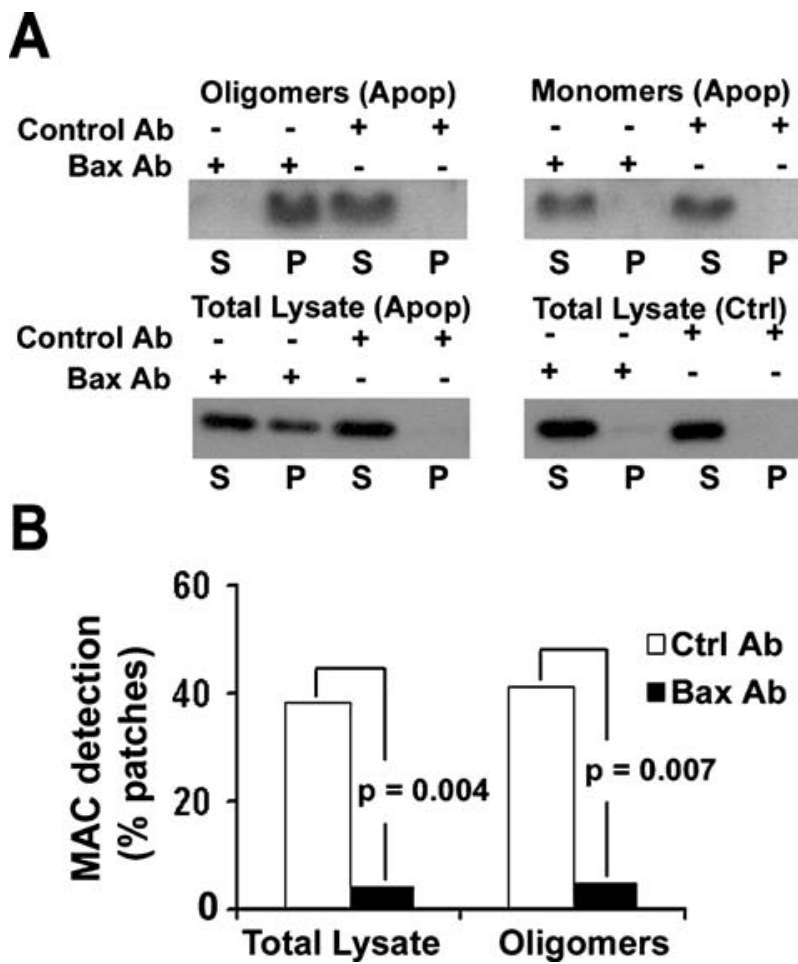

Fig. 3 Bax andlor Bak are components of MAC. (A) Mitochondrial lysates were prepared from apoptotic HeLa cells. Total mitochondrial lysates and chromatographic fractions containing oligomeric and monomeric Bax were examined by Western blotting. The blots show the presence of $\mathrm{Bax}$ in pellets $(\mathrm{P})$ and supernatants $(\mathrm{S})$ after immunoprecipitation with anti-Bax antibodies $(\mathrm{Bax} \mathrm{Ab})$ or total rabbit $\mathrm{IgG}$ (control Ab). (B) The supernatants from total lysates and oligomeric fractions after immunoprecipitation with anti-Bax antibodies (Bax Ab, closed) or with total rabbit IgG (Control Ab, open) as in panel A were reconstituted into proteoliposomes. The histograms show the frequency of detecting MAC in each proteoliposome preparation. MAC was detected by patch clamping. $N=20-23$ independent patches/condition.

investigators and culminated in a series of papers from Mario Zoratti's group [59-65]. In earlier studies, PTP channel activity was referred to as MCC (mitochondrial multiple conductance channel) or MMC (mitochondrial megachannel). Although much smaller than MAC and Bax channels, PTP is a high conductance channel (Table 1). The peak conductance is $\sim 1.2 \mathrm{nS}$ and there are multiple conductance levels. Transitions between sublevels are typically $0.3-1 \mathrm{nS}$. Unlike MAC and Bax channels, this slightly cation selective channel is voltage dependent, closing with matrix positive potentials. Interestingly, while overexpression of Bcl-2 had no detectable effect on the channel activity, this protein suppressed calcium-activation of the PTP in both swelling and electrophysiological experiments [30, 31].

While MAC contains at least Bax and/or Bak, the molecular identity of PTP has not yet been resolved. The adenine nucleotide translocator or ANT was for many years thought to be tightly linked to the PTP because of the abil-
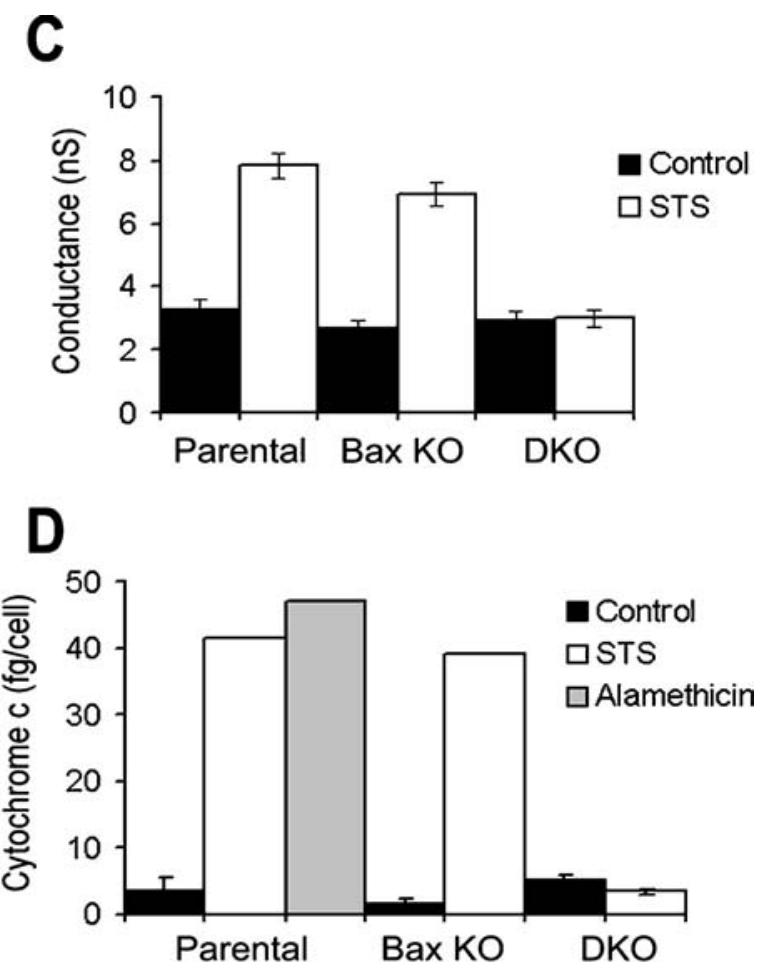

$\mathrm{p}$ values were calculated using the Fisher Exact Statistical Test. (C) Mitochondria were isolated from three MEF cell lines [Parental, single $\mathrm{Bax} \mathrm{KO}$, and $\mathrm{Bax} / \mathrm{Bak}$ double $\mathrm{KO}$ (DKO)] that were (STS) and were not (control) treated with staurosporine. The mean conductance of the outer membrane was measured by patch-clamping isolated mitochondria ( $N=20-23$ patches per condition). (D) An ELISA assay was used to assess cytochrome $c$ release in the supernatants after permeabilization of the cells with digitonin. Alamethicin $(80 \mu \mathrm{g} / \mathrm{mL})$ was added during digitonin treatment as a positive control for cytochrome $c$ release using the method of Polster et al. [102] This figure is reprinted from Fig. 4 of Dejean et al.(2006) Cell Death \& Diff. 13: 1387-95 [10]

ity of Bongkrekic acid to close PTP and inhibit ANT while atractyloside opens PTP and ANT. Hence, it was quite surprising that the PTP remained after the genes responsible for the ANT were knocked out by Wallace's group [66]. They reported that ANT confers effector sensitivity to the PTP, but ANT does not provide the pore for the PTP. However, a more recent report indicates that there is yet a fourth isoform of ANT in liver, testis and brain [67] that is sensitive to both to carboxyatractyloside and bongkrekic acid. While these findings have returned some of the spotlight to ANT's putative role in PTP, the controversy continues. The voltage dependent anion-selective channel or VDAC (also called mitochondrial porin) is another putative component of the PTP in the outer membrane. Molecular studies of VDAC null mice are not possible as this channel is essential for viability. Efforts to generate a knockout of all three isoforms of VDAC in mammalian cells have not been successful and so the role of VDAC in PTP remains unknown [68]. However, studies 


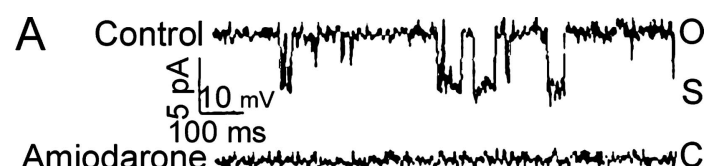

B

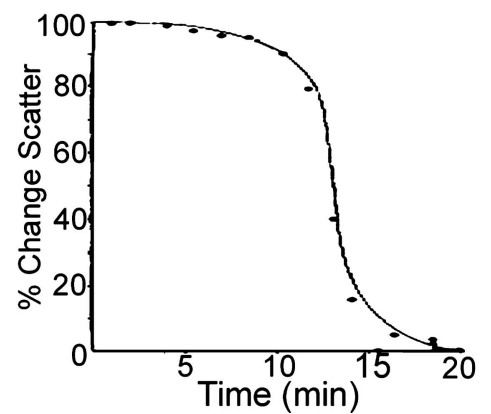

Fig. 4 The PTP is a high conductance channel inhibited by amiodarone. (A) Current traces are shown of a mouse liver mitoplast patch at $10 \mathrm{mV}$ in the absence (control) and presence of $4 \mu \mathrm{M}$ amiodarone. The $1.2 \mathrm{nS}$ peak open $(\mathrm{O}), 550 \mathrm{pS}$ sub- $(\mathrm{S})$ and closed $(\mathrm{C})$ states are indicated. Solution was symmetrical $150 \mathrm{mM}$ KCI $5 \mathrm{mM}$ HEPES $1 \mathrm{mM}$ EGTA $0.95 \mathrm{mM} \mathrm{CaCl}_{2}\left(\sim 10^{-7} \mathrm{M}\right.$ free $\left.\mathrm{Ca}\right) \mathrm{pH}$ 7.4. Modified from Fig. 1 of Antonenko et al. (1991) FEBS Letters 285: 89-93 [88]. (B) The time course of changes in light scattering induced by $10 \mu \mathrm{M}$ calcium in beef heart mitochondria is shown. The light-scattering data at $520 \mathrm{~nm}$ were normalized by calling the initial light-scattering reading $100 \%$ and the final reading $0 \%$. Modified from Fig. 1 of. Hunter and Haworth (1979) Arch. Biochem. Biophys. 195:453-459 [57] with permission from Elsevier

suggest VDAC might have roles in apoptosis as a target of anti-apoptotic proteins and in sequestration of Bak [69-72]. Nevertheless, the lack of a molecular identity for PTP has significantly hampered progress in our understanding of this phenomenon and its putative role in apoptosis.

\section{Does PTP have a role in apoptosis?}

At this time, the role of the PTP in apoptosis is quite controversial. While some studies implicate PTP as an initiating event in the mitochondrial pathway [11, 12, 73-75], others indicate PTP opening is a consequence of the degradation stage of apoptosis. Compounds that induce the permeability transition in isolated mitochondria often cause apoptosis and PTP is blocked or delayed by the anti-apoptotic drug cyclosporine A [64, 76-78]. Suppression of the calciumactivation of the PTP by overexpression of Bcl-2 supports a role for this channel in apoptosis [31, 79]. While some believe PTP plays a major role in the mitochondrial pathway of apoptosis, recent studies show that sustained PTP opening is instead predominantly involved in necrosis and ischemia-reperfusion injury [80-82].

Cyclophilin-D is a regulator of the PTP. Surprisingly, cells deficient in cyclophilin-D die normally after activation of either the extrinsic or intrinsic pathways, but show resistance to necrotic cell death induced by reactive oxygen species and $\mathrm{Ca}^{2+}$ overload [80-82]. These findings strongly indicate that PTP opening is a consequence, rather than an initiator, of apoptosis [83-85]. Nevertheless, the opening of PTP, although subsequent to MAC, may result in a more complete release of cytochrome $c$ and other pro-apoptotic factors from the cristae, which would synergistically amplify the apoptotic cascade initiated by MAC. Furthermore, the delay in the progression of apoptosis caused by PTP inhibitors like cyclosporine A may be related to their ability to delay depolarization and energy production as well as any synergistic effects of further cytochrome $c$ release from the cristae.

\section{Pharmacology of MAC, Bax, and PTP}

The ability to open or close MAC and PTP inside cells can have far reaching therapeutic value and may offer clues as to the underlying mechanisms of apoptosis. MAC and PTP are potential targets for novel therapies since opening MAC and PTP with agonists may induce cell death while preventing their opening with blockers may prevent cell death. Some of the blockers and inducers of PTP and MAC activity are shown in Table 2. Comprehensive lists of PTP effectors have been presented in reviews $[78,86]$. Cyclosporine A has long been used to delay apoptotic death and is a PTP blocker with $\mathrm{nM}$ affinity [11, 76, 77]. However, cyclosporine A does not block MAC channels [27]. While trifluoperazine, dibucaine, and propranolol block both MAC and PTP, there appear to be significant differences in $\mathrm{IC}_{50}$ for the two channels $[27,87$, 88]. More recently, a promising study identified two blockers of Bax channels, i.e., MAC [47] (Fig. 5). These blockers, bci1 and bci2, prevented cytochrome $c$ release in vitro and in vivo and protected neurons in a mice animal model of brain ischemia [47]. This and other studies indicate that MAC and perhaps PTP are potential therapeutic targets to reduce tissue damage associated with ischemic events, i.e. infarction and stroke. Unfortunately, many agents affecting PTP were identified prior to characterization of MAC. Hence, their ability to prevent cytochrome $c$ release may need to be reevaluated in light of the integral role of MAC in this process. Future studies could perhaps rely on the findings that the mitochondrial swelling and depolarization associated with PTP opening are not thought to be associated with MAC formation.

Cytochrome $c$ and RNAase A have been shown to reversibly and transiently reduce the conductance through MAC. However, the effects of these proteins demonstrate that they partition into the pore and are translocated as has been found with DNA or ATP moving into Hemolysin A channels or VDAC, respectively [89, 90]. These effects are distinct from those found with compounds like dibucaine 
Fig. 5 Structures of the Bax channel inhibitors and inhibition of Bax

channel-forming activity in liposomes. (A) the structures of Bci1 and Bci2 are shown. (B-E) liposomes containing $20 \mathrm{mM}$ 5,6-carboxyfluorescein were diluted to $1 \mathrm{ml}$ in PBS.

Oligomeric Bax was

pre-incubated with increasing concentrations of Bci1 or Bci2 at room temperature for $1 \mathrm{~min}$. B and $\mathrm{D}$, at time 0 , liposomes were added to the Bax/compound mixture, and the change in fluorescence (excitation at $488 \mathrm{~nm}$ and emission at $520 \mathrm{~nm}$ ) was recorded every $3 \mathrm{~s}$ for

$3 \mathrm{~min}$. $\mathrm{C}$ and $\mathrm{E}$, the fluorescence values at $t=120 \mathrm{~s}$ were plotted against the log compound concentration, and the IC50 values were determined. The IC50 values were determined to be $0.81 \pm 0.22 \mu \mathrm{M}(n=15)$ for Bci1 and $0.89 \pm 0.29 \mu \mathrm{M}$ $(n=10)$ for Bci2. AU, arbitrary units. Figure from Hetz et al., 2005, J.Biol. Chem. 280: 42960-42970 [47]

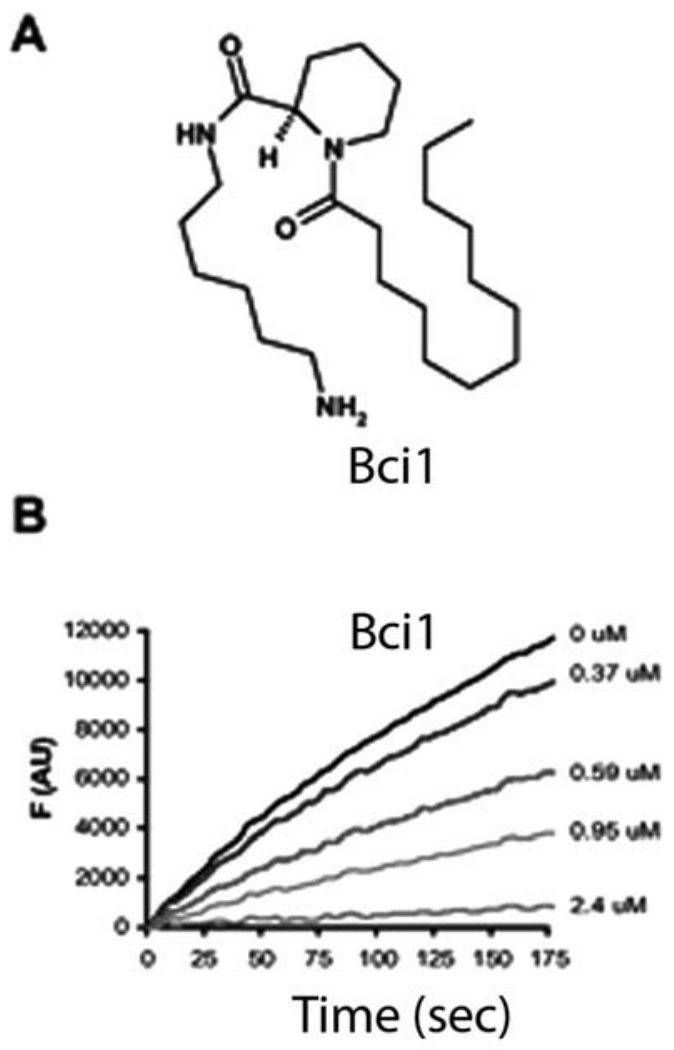

D

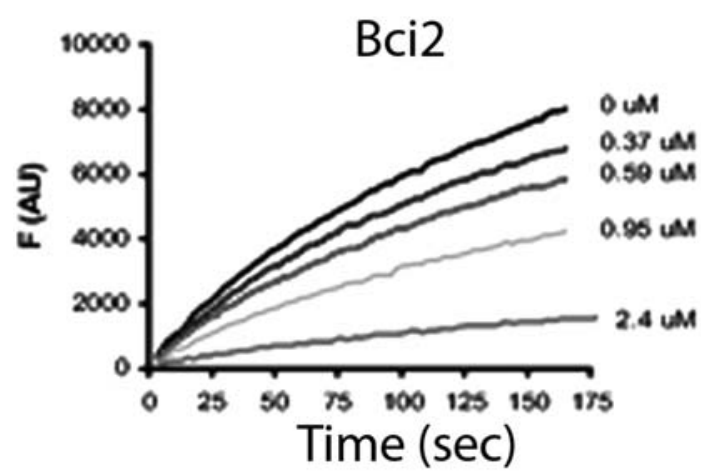

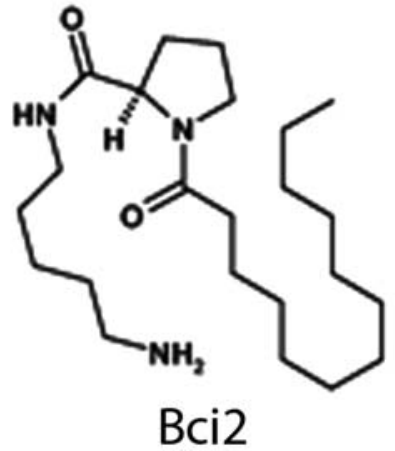

C

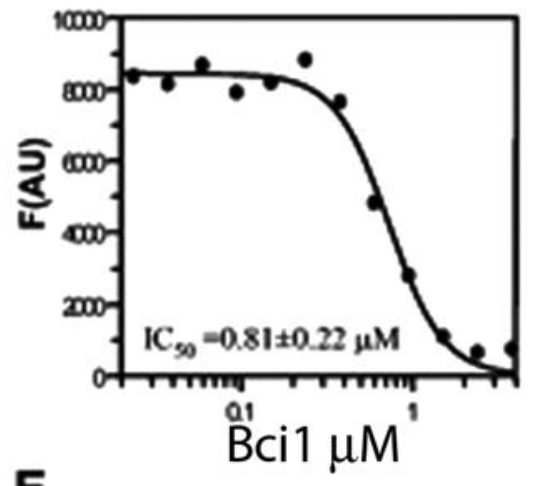

E

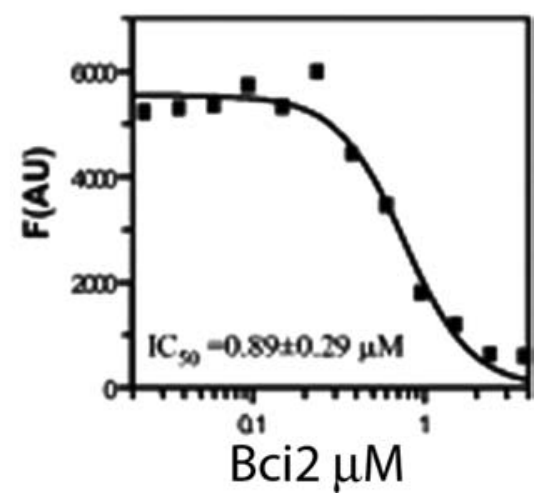

[27], bci1 [47], or cyclosporine A [77] which could have potential therapeutic value.

\section{MAC or PTP?}

How can one determine whether mitochondrial apoptosis is due to the opening of MAC or PTP? Activation of both pores release cytochrome $c$. The two pores differ in their effects on the mitochondrial membrane potential and the ability to make ATP. Opening PTP depolarizes mitochondria and uncouples oxidative phosphorylation, while MAC, at least initially, does not. MAC was discovered by patch clamping mitochondria of FL5. 12 cells twelve hours after interleukin-
3 withdrawal. At this time, the mitochondria still maintain their membrane potential as indicated by Rhodamine 123 staining. However, the mitochondria are depolarized by eighteen hours (unpublished data of Kinnally). These data indicate that MAC formation occurred in the absence of PTP opening in these cells after IL-3 withdrawal. There have been several reports that release of cytochrome $c$ occurs prior to depolarization [14]. Hence, these events are not necessarily synchronized. Cytochrome $c$ release corresponds to MAC formation when release is not accompanied by mitochondrial depolarization. However, the mechanism of release is less clear if the onset of cytochrome $c$ release cannot be resolved from depolarization, as depletion of cytochrome $c$ from the electron transport network OR opening of PTP may underlie 
mitochondrial depolarization [91]. Alternatively, the two channels can be distinguished pharmacologically by the ability of cyclosporine A to block PTP and bcil to block MAC [47, 76].

\section{Apoptosis in disease}

Although apoptosis is an essential process for all multicellular organisms, it is also involved or associated with a wide range of diseases and pathological conditions [9297]. This has made apoptosis an attractive potential target for treatment of various diseases. Deregulation of the apoptosis processes can lead to either an inappropriate increase in apoptosis, associated with diseases such as ischemia and neurodegenerative pathologies, or to impaired apoptosis, frequently associated with various cancers and autoimmune diseases. Thus, regulation of apoptosis in these pathologies could be beneficial. However, several issues need to be addressed before a successful regulation of apoptosis can be implemented for treatment of diseases.

As mentioned above, apoptosis is an essential physiological process and a too broad disturbance of this process will inevitably result in severe side effects. Early on, the executioner caspases were envisioned as crucial anti-apoptotic targets, since several apoptosis signaling cascades converge at this point. Hence, it was thought that it would not be necessary to know which apoptosis signaling cascade was involved in the disease process. Such attempts failed, due to toxicity and severe side effects, presumably due to inhibition of essential apoptotic processes. A main problem in treating diseases is that the apoptosis pathway involved in a specific pathology is often not known. In order to be successful and minimize undesired side effects and toxicity, the intervention needs to be targeted as specifically as possible in order to inhibit or activate just the pathway involved in the disease process. In general, this means that we should intervene as upstream in the pathway as possible. For example, if we want to prevent apoptosis mediated through the mitochondrial pathway, the best point of intervention would presumably be at the activation step of Bax and/or Bak, before the proteins get activated and perturb mitochondrial function through their oligomerization in the membrane. This would restrict perturbation of apoptosis signaling in the cells, affecting only the specific activation signal, and leave other $\mathrm{Bax} / \mathrm{Bak}$ activating processes functional. It would also retain fully functional mitochondria in the cells, which might be extremely important.

In most pathological conditions, apoptosis is not the direct cause of the disease. Hence, it is difficult to predict the effect that inhibition of apoptosis will have in the disease process. Inhibition of apoptosis could limit or prevent damage and tissue destruction, which should favor recovery and reduce impairments. In several diseases cell death is not exclusively by apoptosis. Necrosis is often also involved and the border between the two processes is not very well defined. One could also envision that cells may enter the apoptosis cascade but then ultimately die by necrosis because of changing conditions [98]. Thus, any apoptosis treatment must also ensure that cells are not just switched to a pathway of necrotic death, which may, in the end, aggravate instead of ameliorate the condition. It is also obvious that activation of programmed cell death must be focused to the target cells in diseases with impaired apoptosis in order to minimize side effects by not inducing inappropriate apoptosis in other cells and tissues.

\section{Conclusions}

Mitochondria provide the gateway through which apoptosis can be executed in a rapid and robust manner. There are two pathways by which cytochrome $c$ can be released from mitochondria to commit the cell to die; activation of either MAC in the outer membrane or PTP in the inner membrane can effectuate this event. At the present time, the evidence indicates that MAC, which is exquisitely regulated by Bcl-2 family proteins, is a principal means of commitment to apoptosis. Activation of the PTP may in fact be a consequence of apoptosis, but its activation may ensure complete execution of the cell. Nevertheless, both PTP and MAC remain potential therapeutic targets which should be exploited to induce cell death in malignancies and prevent cell death in degenerative and ischemia-associated pathologies.

Acknowledgments This work supported in part by NIH grant GM57249 and NSF grants MCB-0235834 to KWK. We thank Laurent Dejean and Sonia Martinez-Caballero (NYUCD) for their insightful discussions.

\section{References}

1. Antonsson B (2004) Mitochondria and the Bcl-2 family proteins in apoptosis signaling pathways. Mol Cell Biochem 256-257:141-155

2. Danial NN, Korsmeyer SJ (2004) Cell death: critical control points. Cell 116:205-219

3. Dejean LM, Martinez-Caballero S, Manon S, Kinnally KW (2006) Regulation of the mitochondrial apoptosis-induced channel, MAC, by BCL-2 family proteins. Biochim Biophys Acta 1762:191-201

4. Fadeel B, Orrenius S (2005) Apoptosis: a basic biological phenomenon with wide-ranging implications in human disease. J Intern Med 258:479-517

5. Green DR, Kroemer G (2004) The pathophysiology of mitochondrial cell death. Science 305:626-629

6. Kuwana T, Newmeyer DD (2003) Bcl-2-family proteins and the role of mitochondria in apoptosis. Curr Opin Cell Biol $15: 691-699$ 
7. Lucken-Ardjomande S, Martinou JC (2005) Regulation of Bcl-2 proteins and of the permeability of the outer mitochondrial membrane. C R Biol 328:616-631

8. Martinez-Caballero S, Dejean LM, Jonas EA, Kinnally KW (2005) The role of the mitochondrial apoptosis induced channel MAC in cytochrome $c$ release. J Bioenerg Biomembr 37:155-164

9. Sharpe JC, Arnoult D, Youle RJ (2004) Control of mitochondrial permeability by Bcl-2 family members. Biochim Biophys Acta 1644:107-113

10. Dejean LM, Martinez-Caballero S, Kinnally KW (2006) Is MAC the knife that cuts cytochrome $c$ from mitochondria during apoptosis? Cell Death Differ 13:1387-1395

11. Bernardi P, Krauskopf A, Basso E et al (2006) The mitochondrial permeability transition from in vitro artifact to disease target. Febs J 273:2077-2099

12. Forte M, Bernardi P (2006) The permeability transition and BCL2 family proteins in apoptosis: co-conspirators or independent agents? Cell Death Differ 13:1287-1290

13. Schmitz I, Kirchhoff S, Krammer PH (2000) Regulation of death receptor-mediated apoptosis pathways. Int J Biochem Cell Biol 32:1123-1136

14. Liu X, Kim CN, Yang J, Jemmerson R, Wang X (1996) Induction of apoptotic program in cell-free extracts: requirement for dATP and cytochrome $c$. Cell 86:147-157

15. Adrain C, Martin SJ (2001) The mitochondrial apoptosome: a killer unleashed by the cytochrome seas. Trends Biochem Sci 26:390-397

16. Daugas E, Susin SA, Zamzami N et al (2000) Mitochondrionuclear translocation of AIF in apoptosis and necrosis. Faseb J 14:729-739

17. Li LY, Luo X, Wang X (2001) Endonuclease G is an apoptotic DNase when released from mitochondria. Nature 412:95-99

18. Li H, Zhu H, Xu CJ, Yuan J (1998) Cleavage of BID by caspase 8 mediates the mitochondrial damage in the Fas pathway of apoptosis. Cell 94:491-501

19. Kroemer G, Reed JC (2000) Mitochondrial control of cell death. Nat Med 6:513-519

20. Marzo I, Brenner C, Zamzami N et al (1998) The permeability transition pore complex: a target for apoptosis regulation by caspases and bcl-2-related proteins. J Exp Med 187:1261-1271

21. Skulachev VP (1996) Why are mitochondria involved in apoptosis? Permeability transition pores and apoptosis as selective mechanisms to eliminate superoxide-producing mitochondria and cell. FEBS Lett 397:7-10

22. De Giorgi F, Lartigue L, Bauer MK et al (2002) The permeability transition pore signals apoptosis by directing Bax translocation and multimerization. Faseb J 16:607-609

23. Dejean LM, Martinez-Caballero S, Guo L et al (2005) Oligomeric Bax is a component of the putative cytochrome $c$ Release Channel MAC, Mitochondrial Apoptosis-induced Channel. Mol Biol Cell 16:2424-2432

24. Guo L, Pietkiewicz D, Pavlov EV et al (2004) Effects of cytochrome $c$ on the mitochondrial apoptosis-induced channel MAC. Am J Physiol Cell Physiol 286:C1109-C1117

25. Pavlov EV, Priault M, Pietkiewicz D et al (2001) A novel, high conductance channel of mitochondria linked to apoptosis in mammalian cells and Bax expression in yeast. J Cell Biol 155:725731

26. Wei MC, Zong WX, Cheng EH et al (2001) Proapoptotic BAX and BAK: a requisite gateway to mitochondrial dysfunction and death. Science 292:727-730

27. Martinez-Caballero S, Dejean LM, Kinnally KW (2004) Some amphiphilic cations block the mitochondrial apoptosis-induced channel, MAC. FEBS Lett 568:35-38

28. Antonsson B, Montessuit S, Sanchez B, Martinou JC (2001) Bax is present as a high molecular weight oligomer/complex in the mitochondrial membrane of apoptotic cells. J Biol Chem 276:11615-11623

29. Guihard G, Bellot G, Moreau C et al (2004) The mitochondrial apoptosis-induced channel (MAC) corresponds to a late apoptotic event. J Biol Chem 279:46542-46550

30. Murphy RC, Schneider E, Kinnally KW (2001) Overexpression of Bcl-2 suppresses the calcium activation of a mitochondrial megachannel. FEBS Lett 497:73-76

31. Murphy AN, Bredesen DE, Cortopassi G, Wang E, Fiskum G (1996) Bcl-2 potentiates the maximal calcium uptake capacity of neural cell mitochondria. Proc Natl Acad Sci USA 93:9893-9898

32. McDonnell JM, Fushman D, Milliman CL, Korsmeyer SJ, Cowburn D (1999) Solution structure of the proapoptotic molecule BID: a structural basis for apoptotic agonists and antagonists. Cell 96:625-634

33. Petros AM, Medek A, Nettesheim DG et al (2001) Solution structure of the antiapoptotic protein bcl-2. Proc Natl Acad Sci USA 98:3012-3017

34. Suzuki M, Youle RJ, Tjandra N (2000) Structure of Bax: coregulation of dimer formation and intracellular localization. Cell 103:645-654

35. Antonsson B, Conti F, Ciavatta A et al (1997) Inhibition of Bax channel-forming activity by Bcl-2. Science 277:370-372

36. Minn AJ, Velez P, Schendel SL et al (1997) Bcl-x(L) forms an ion channel in synthetic lipid membranes. Nature 385:353-357

37. Schlesinger PH, Gross A, Yin XM et al (1997) Comparison of the ion channel characteristics of proapoptotic BAX and antiapoptotic BCL-2. Proc Natl Acad Sci USA 94:11357-11362

38. Hsu YT, Youle RJ (1998) Bax in murine thymus is a soluble monomeric protein that displays differential detergent-induced conformations. J Biol Chem 273:10777-10783

39. Antonsson B, Montessuit S, Lauper S, Eskes R, Martinou JC (2000) Bax oligomerization is required for channel-forming activity in liposomes and to trigger cytochrome $c$ release from mitochondria. Biochem J 345(Pt 2):271-278

40. Desagher S, Osen-Sand A, Nichols A et al (1999) Bid-induced conformational change of $\mathrm{Bax}$ is responsible for mitochondrial cytochrome $c$ release during apoptosis. J Cell Biol 144:891-901

41. Nechushtan A, Smith CL, Hsu YT, Youle RJ (1999) Conformation of the Bax C-terminus regulates subcellular location and cell death. Embo J 18:2330-2341

42. Roucou X, Rostovtseva T, Montessuit S, Martinou JC, Antonsson B (2002) Bid induces cytochrome $c$-impermeable Bax channels in liposomes. Biochem J 363:547-552

43. Chan SK, Tulloss I, Margoliash E (1966) Primary structure of the cytochrome $c$ from the snapping turtle, Chelydra serpentina. Biochemistry 5:2586-2597

44. Saito M, Korsmeyer SJ, Schlesinger PH (2000) BAX-dependent transport of cytochrome $c$ reconstituted in pure liposomes. Nat Cell Biol 2:553-555

45. Griffiths GJ, Dubrez L, Morgan CP et al (1999) Cell damageinduced conformational changes of the pro-apoptotic protein Bak in vivo precede the onset of apoptosis. J Cell Biol 144:903-914

46. Martinou J, Youle R (2006) Which came first, the cytochrome $c$ release or the mitochondrial fission? Cell Death Differ 13:1291-1295

47. Hetz C, Vitte PA, Bombrun A et al (2005) Bax channel inhibitors prevent mitochondrion-mediated apoptosis and protect neurons in a model of global brain ischemia. J Biol Chem 280:42960-42970

48. Brustovetsky T, Antonsson B, Jemmerson R, Dubinsky JM, Brustovetsky N (2005) Activation of calcium-independent phospholipase A2 (iPLA2) in brain mitochondria and release of apoptogenic factors by BAX and truncated BID. J Neurochem 94:980-994

49. Adrain C, Creagh EM, Martin SJ (2001) Apoptosis-associated release of Smac/DIABLO from mitochondria requires active caspases and is blocked by Bcl-2. Embo J 20:6627-6636 
50. Arnoult D, Gaume B, Karbowski M, Sharpe JC, Cecconi F, Youle RJ (2003) Mitochondrial release of AIF and EndoG requires caspase activation downstream of Bax/Bak-mediated permeabilization. Embo J 22:4385-4399

51. Munoz-Pinedo C, Guio-Carrion A, Goldstein JC, Fitzgerald P, Newmeyer DD, Green DR (2006) Different mitochondrial intermembrane space proteins are released during apoptosis in a manner that is coordinately initiated but can vary in duration. Proc Natl Acad Sci USA 103:11573-11578

52. Stoica BA, Movsesyan VA, Knoblach SM, Faden AI (2005) Ceramide induces neuronal apoptosis through mitogen-activated protein kinases and causes release of multiple mitochondrial proteins. Mol Cell Neurosci 29:355-371

53. Siskind LJ, Kolesnick RN, Colombini M (2002) Ceramide channels increase the permeability of the mitochondrial outer membrane to small proteins. J Biol Chem 277:26796-26803

54. Mikhailov V, Mikhailov M, Pulkrabek DJ, Dong Z, Venkatachalam MA, Saikumar P (2001) Bcl-2 prevents bax oligomerization in the mitochondrial outer membrane. J Biol Chem 276:1836118374

55. Haworth RA, Hunter DR (1979) The $\mathrm{Ca}^{2+}$-induced membrane transition in mitochondria. II. Nature of the $\mathrm{Ca}^{2+}$ trigger site. Arch Biochem Biophys 195:460-467

56. Haworth S, Hunter J (2004) Should the RCN lose its trade union function? Nurs Times 100:18

57. Hunter DR, Haworth RA (1979) The Ca2+-induced membrane transition in mitochondria. I. The protective mechanisms. Arch Biochem Biophys 195:453-459

58. Hunter DR, Haworth RA (1979) The Ca2+-induced membrane transition in mitochondria. III. Transitional $\mathrm{Ca} 2+$ release. Arch Biochem Biophys 195:468-477

59. Kinnally KW, Campo ML, Tedeschi H (1989) Mitochondrial channel activity studied by patch-clamping mitoplasts. J Bioenerg Biomembr 21:497-506

60. Kinnally KW, Zorov D, Antonenko Y, Perini S (1991) Calcium modulation of mitochondrial inner membrane channel activity. Biochem Biophys Res Commun 176:1183-1188

61. Bernardi P, Vassanelli S, Veronese P, Colonna R, Szabo I, Zoratti M (1992) Modulation of the mitochondrial permeability transition pore. Effect of protons and divalent cations. J Biol Chem 267:2934-2939

62. Petronilli V, Szabo I, Zoratti M (1989) The inner mitochondrial membrane contains ion-conducting channels similar to those found in bacteria. FEBS Lett 259:137-143

63. Szabo I, Bernardi P, Zoratti M (1992) Modulation of the mitochondrial megachannel by divalent cations and protons. $\mathrm{J}$ Biol Chem 267:2940-2946

64. Szabo I, Zoratti M (1991) The giant channel of the inner mitochondrial membrane is inhibited by cyclosporin A. J Biol Chem 266:3376-3379

65. Szabo I, Zoratti M (1992) The mitochondrial megachannel is the permeability transition pore. J Bioenerg Biomembr 24:111-117

66. Kokoszka J, Waymire K, Levy S et al (2004) The ADP/ATP translocator is not essential for the mitochondrial permeability transition pore. Nature 427:461-465

67. Dolce V, Scarcia P, Iacopetta D, Palmieri F (2005) A fourth ADP/ATP carrier isoform in man: identification, bacterial expression, functional characterization and tissue distribution. FEBS Lett 579:633-637

68. Wu S, Sampson M, Decker W, Craigen W (1999) Each mammalian mitochondrial outer membrane porin protein is dispensable: effects on cellular respiration. Biochim Biophys Acta 1452:68-78

69. Cheng EH, Sheiko TV, Fisher JK, Craigen WJ, Korsmeyer SJ (2003) VDAC2 inhibits BAK activation and mitochondrial apoptosis. Science 301:513-517
70. Vander Heiden MG, Chandel NS, Li XX, Schumacker PT, Colombini M, Thompson CB (2000) Outer mitochondrial membrane permeability can regulate coupled respiration and cell survival. Proc Natl Acad Sci USA 97:4666-4671

71. Vander Heiden MG, Li XX, Gottleib E, Hill RB, Thompson CB, Colombini M (2001) Bcl-xL promotes the open configuration of the voltage-dependent anion channel and metabolite passage through the outer mitochondrial membrane. J Biol Chem 276:19414-19419

72. Rostovtseva TK, Antonsson B, Suzuki M, Youle RJ, Colombini M, Bezrukov SM (2004) Bid, but not Bax, regulates VDAC channels. J Biol Chem 279:13575-13583

73. Susin SA, Lorenzo HK, Zamzami N et al (1999) Mitochondrial release of caspase- 2 and -9 during the apoptotic process. J Exp Med 189:381-394

74. Susin SA, Lorenzo HK, Zamzami N et al (1999) Molecular characterization of mitochondrial apoptosis-inducing factor. Nature 397:441-446

75. Susin SA, Zamzami N, Castedo M et al (1996) Bcl-2 inhibits the mitochondrial release of an apoptogenic protease. J Exp Med 184:1331-1341

76. Broekemeier K, Pfeiffer D (1989) Cyclosporin A-sensitive and insensitive mechanisms produce the permeability transition in mitochondria. Biochem Biophys Res Commun 163:561-566

77. Broekemeier KM, Carpenter-Deyo L, Reed DJ, Pfeiffer DR (1992) Cyclosporin A protects hepatocytes subjected to high $\mathrm{Ca}^{2+}$ and oxidative stress. FEBS Lett 304:192-194

78. Gunter T, Pfeiffer D (1990) Mechanisms by which mitochondria transport calcium. Am J Physiol 258:C755-C786

79. Murphy RC, Diwan JJ, King M, Kinnally KW (1998) Two high conductance channels of the mitochondrial inner membrane are independent of the human mitochondrial genome. FEBS Lett 425:259-262

80. Nakagawa T, Shimizu S, Watanabe T et al (2005) Cyclophilin D-dependent mitochondrial permeability transition regulates some necrotic but not apoptotic cell death. Nature 434:652-658

81. Baines CP, Kaiser RA, Purcell NH et al (2005) Loss of cyclophilin D reveals a critical role for mitochondrial permeability transition in cell death. Nature 434:658-662

82. Basso E, Fante L, Fowlkes J, Petronilli V, Forte MA, Bernardi P (2005) Properties of the permeability transition pore in mitochondria devoid of Cyclophilin D. J Biol Chem 280:18558-18561

83. Halestrap AP (2006) Calcium, mitochondria and reperfusion injury: a pore way to die. Biochem Soc Trans 34:232-237

84. Halestrap A (2005) Biochemistry: a pore way to die. Nature 434:578-579

85. Halestrap AP (2004) Mitochondrial permeability: dual role for the ADP/ATP translocator? Nature 430:1 p following 983

86. Zoratti M, Szabo I (1995) The mitochondrial permeability transition. Biochimica et Biophysica Acta 1241:139-176

87. Hoyt KR, Sharma TA, Reynolds IJ (1997) Trifluoperazine and dibucaine-induced inhibition of glutamate-induced mitochondrial depolarization in rat cultured forebrain neurones. $\mathrm{Br} \mathrm{J}$ Pharmacol 122:803-808

88. Antonenko YN, Kinnally KW, Perini S, Tedeschi H (1991) Selective effect of inhibitors on inner mitochondrial membrane channels. FEBS Lett 285:89-93

89. Kasianowicz JJ, Henrickson SE, Weetall HH, Robertson B (2001) Simultaneous multianalyte detection with a nanometer-scale pore. Anal Chem 73:2268-2272

90. Rostovtseva T, Colombini M (1997) VDAC channels mediate and gate the flow of ATP: implications for the regulation of mitochondrial function. Biophys J 72:1954-1962

91. Heiskanen KM, Bhat MB, Wang HW, Ma J, Nieminen AL (1999) Mitochondrial depolarization accompanies cytochrome $c$ release during apoptosis in PC6 cells. J Biol Chem 274:5654-5658 
92. Chandra J, Zhivotovsky B, Zaitsev S, Juntti-Berggren L, Berggren PO, Orrenius S (2001) Role of apoptosis in pancreatic beta-cell death in diabetes. Diabetes 50(Suppl 1):S44S47

93. Kockx MM, Knaapen MW (2000) The role of apoptosis in vascular disease. J Pathol 190:267-280

94. Krajewski S, Blomqvist C, Franssila K et al (1995) Reduced expression of proapoptotic gene BAX is associated with poor response rates to combination chemotherapy and shorter survival in women with metastatic breast adenocarcinoma. Cancer Res 55:4471-4478

95. Krammer PH (2000) CD95's deadly mission in the immune system. Nature 407:789-795

96. Yuan J, Yankner BA (2000) Apoptosis in the nervous system. Nature 407:802-809

97. Reed JC (1998) Dysregulation of apoptosis in cancer. Cancer J. Sci. Am 4:S8-S14

98. Nicotera P, Leist M, Ferrando-May E (1999) Apoptosis and necrosis: different execution of the same death. Biochem Soc Symp 66:69-73

99. Hille B (2001) Ionic channels of excitable membranes. Sinauer Assoc, Sunderland, MA, pp 351-352

100. Bombrun A, Gerber P, Casi G, Terradillos O, Antonsson B, Halazy S (2003) 3,6-dibromocarbazole piperazine derivatives of 2-propanol as first inhibitors of cytochrome $c$ release via Bax channel modulation. J Med Chem 46: 4365-4368

101. Kinnally KW, Zorov DB, Antonenko YN, Snyder SH, McEnery MW, Tedeschi H (1993) Mitochondrial benzodiazepine receptor linked to inner membrane ion channels by nanomolar actions of ligands. Proc Natl Acad Sci USA 90: 1374-1378

102. Polster BM, Basanez G, Young M, Suzuki M, Fiskum G (2003) Inhibition of Bax-induced cytochrome $c$ release from neural cell and brain mitochondria by dibucaine and propranolol. J Neurosci 23:2735-27343 